\title{
11 Abbildungsverzeichnis
}

Abb. 1 Beispiel Fragebogen Teil $1-22$

Abb. 2 Beispiel Fragebogen Teil $2-22$

Abb. 3 Wortprofil zu ziehen, Screen-Ausschnitt (DWDS_WP_ziehen; 28.05.2018) — 97

Abb. 4 Kookkurrenzen und signifikante Nachbarn zu akzeptieren, Screen-Ausschnitt (WoSchaPo_akzeptieren; 31.05.2018) -103

Abb. 5 Verwendungsbeispiele zu akzeptieren, Screen-Ausschnitt (WoSchaPo_akzeptieren; 31.05.2018) 104

Abb. 6 Wortformenliste zu \&akzeptieren, Screen-Ausschnitt (COSMAS-II) - 107

Abb. 7 KWIC zu \&akzeptieren, Screen-Ausschnitt (COSMAS-II) $\mathbf{1 0 8}$

Abb. 8 Kookkurrenzprofil zu \&akzeptieren, Screen-Ausschnitt (COSMAS-II) 109

Abb. 9 Ergebnisübersicht der Kookkurrenzanalyse zu akzeptieren —-113

Abb. 10 Schlagwortwolke, Screen-Ausschnitt (DWDS_WP_akzeptieren; 31.05.2018) 116

Abb. 11 Tabellarische Übersicht, Screen-Ausschnitt (DWDS_WP_akzeptieren; 31.05.2018) - 117

Abb. 12 Einzelbelege zu Entscheidung akzeptieren, Screen-Ausschnitt (DWDS_WP_ akzeptieren; 31.05.2018) - 117

Abb. 13 Beispiel Fragebogen (Teil 2) 123

Abb. 14 Testitem für das Verb akzeptieren - 138

Abb. 15 Auszug Fragebogen $(035 \mathrm{IN})-143$

Abb. 16 Auszug Fragebogen (033EM) - 143

Abb. 17 DUDEN $\left(2012^{4}\right)$ geben (Hervorhebung durch Kasten ergänzt, TvdB) 173

Abb.18 Google_WB_geben; 04.06.2018 (Hervorhebung durch Kasten ergänzt, TvdB) $\mathbf{1 7 5}$

Abb. 19 Beispielitem zu trinken - 190

Abb. $\mathbf{2 0}$ Testitem sich baden - 211

Abb. 21 Testitem baden $-\mathbf{2 1 2}$

Abb. 22 Testitem sich duschen $\mathbf{2 2 0}$

Abb. 23 Testitem duschen $-\mathbf{2 2 1}$ 
